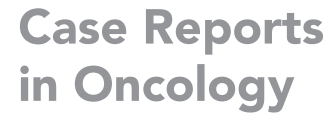

\title{
Role of Immunotherapy in Pulmonary Angiosarcoma: A Case Report
}

\author{
Quang Tien Nguyen ${ }^{a} \quad$ Anh Tuan Pham ${ }^{a}$ Thuy Thi Nguyen ${ }^{a}$ \\ Tam Thi Thanh Nguyen ${ }^{a}$ Ky Van Le ${ }^{b}$ \\ ${ }^{a}$ Department of Medical Oncology, National Cancer Hospital, Hanoi, Vietnam; ${ }^{b}$ Department \\ of Pathology, National Cancer Hospital, Hanoi, Vietnam
}

\section{Keywords}

Pulmonary angiosarcoma $\cdot$ Immunotherapy $\cdot$ Prembrolizumab

\section{Abstract}

Pulmonary angiosarcoma is a rare clinical entity with a poor prognosis and no established therapeutic strategies. We present the first case to our knowledge of metastatic pulmonary angiosarcoma, treated with checkpoint inhibitor immunotherapy, and have an excellent response. Until now, patient has been treated with immunotherapy for 1 year, and his disease is stable and well-tolerated.

\section{Introduction}

Pulmonary angiosarcoma is a rare malignancy that accounts for $0.001-0.03 \%$ of all cancer [1]. Pulmonary angiosarcoma is associated with a very aggressive clinical course and a poor prognosis [2]. Because of this rare entity, treatment options have not been well-established. Systemic chemotherapy is used in metastatic setting, but it is short-lived and its benefit for patients who progress after first line is unclear. Here, we present a case of metastatic pulmonary angiosarcoma that have an excellent response to immunotherapy after progression on first line chemotherapy.

\section{Case Presentation}

A 64-year-old male with 30-pack-year smoking history presented with cough and mild hemoptysis. Further, he complained of progressive dyspnea with a decline in his exercise tolerance. He also had a significant weight loss of $4 \mathrm{~kg}$ for 3 months period. He had no personal

\section{Karger!}


or family history of respiratory diseases. Physical examination demonstrated no significant abnormalities.

Bronchoscopy revealed that the tumor completely obstructed the left lower lobe bronchus and bleeding easily. A rigid bronchoscopic biopsy was performed. Immunohistochemically, tumor cells stained positive for CD31, ERG, Vimentin (Fig. 1), and while CD34 negative. Pulmonary sample was reviewed by many pathologists and revealed an angiosarcoma.

Computed tomography scans identified a left hilum lung mass with enlargement of mediastinal and right hilum lymph nodes (Fig. 2a). Bone scan showed an increased uptake in left second rib with high suspicion of metastasis (Fig. 2b). Magnetic resonance imaging of brain was normal. We therefore diagnosed stage IV pulmonary angiosarcoma.

Singer agent chemotherapy with paclitaxel was initiated due to poor patient performance status. After 3 cycles, the patient exhibited radiographic progression in all known lesions. The left perihilar mass increased rapidly causing a complete atelectasis of the same side (Fig. 3a). Clinically, patient's symptoms such as cough, chest pain, and dyspnea became more intense. His performance status is poorer. We thus had difficulties to intruder a second chemotherapy. As his tumor was found to express PD-L1 (TPS 25\%), patient was started on pembrolizumab $2 \mathrm{mg} / \mathrm{kg}$ every 21 days. During the next 9 weeks, the clinical symptoms of the patient significantly improved, and we obtained an excellent response on lung tumor (Fig. 3b). To date, patient has completed a total of 19 cycles of pembrolizumab. After 1 year of treatment with immunotherapy, he has a stable disease and tolerated well.

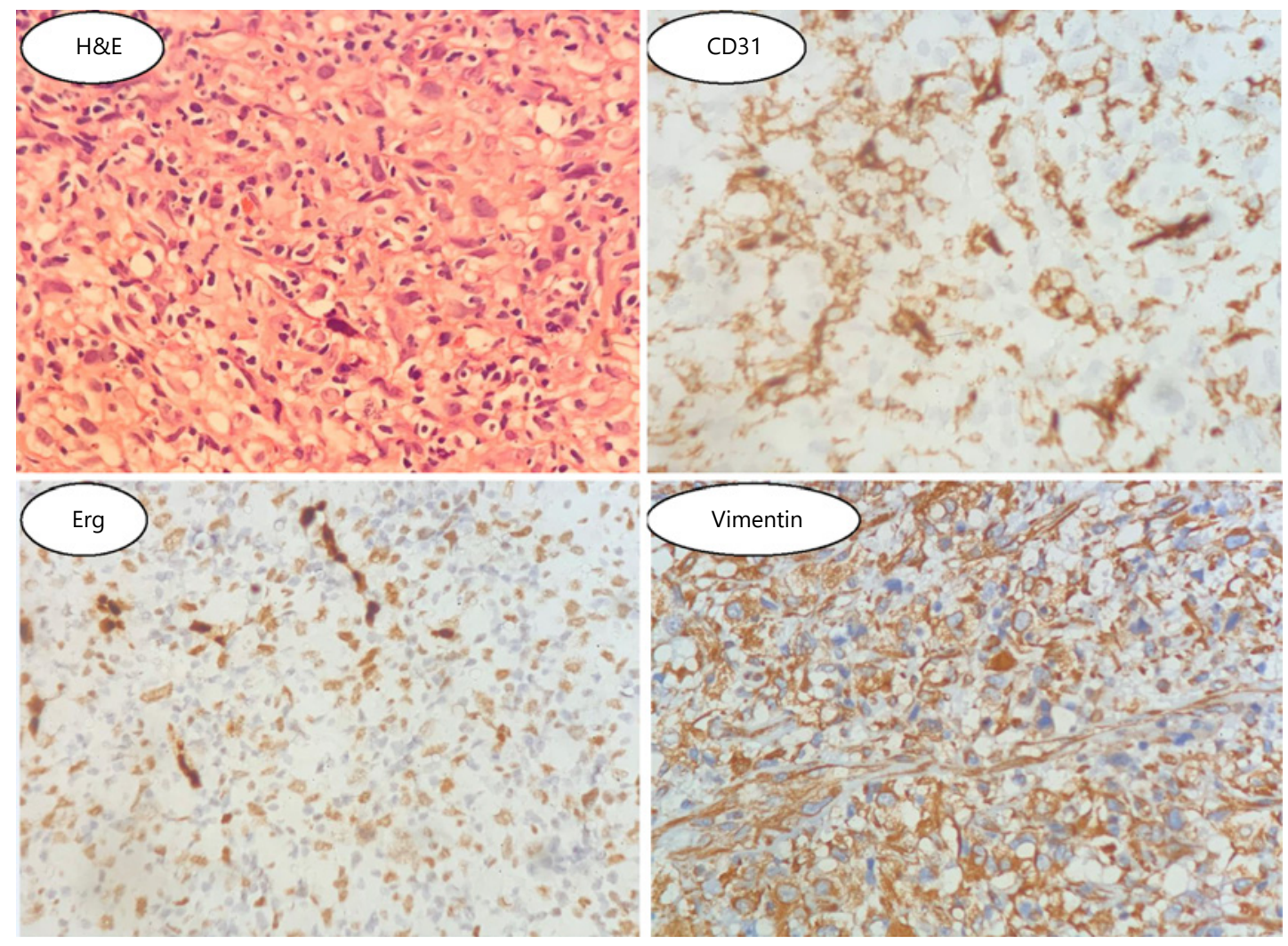

Fig. 1. Representative pathologic sections of pulmonary angiosarcoma. H and E stain and immunohistochemistry revealed that the tumor cells were positive for CD31, ERG, and Vimentin. 


\section{Case Reports in Oncology}
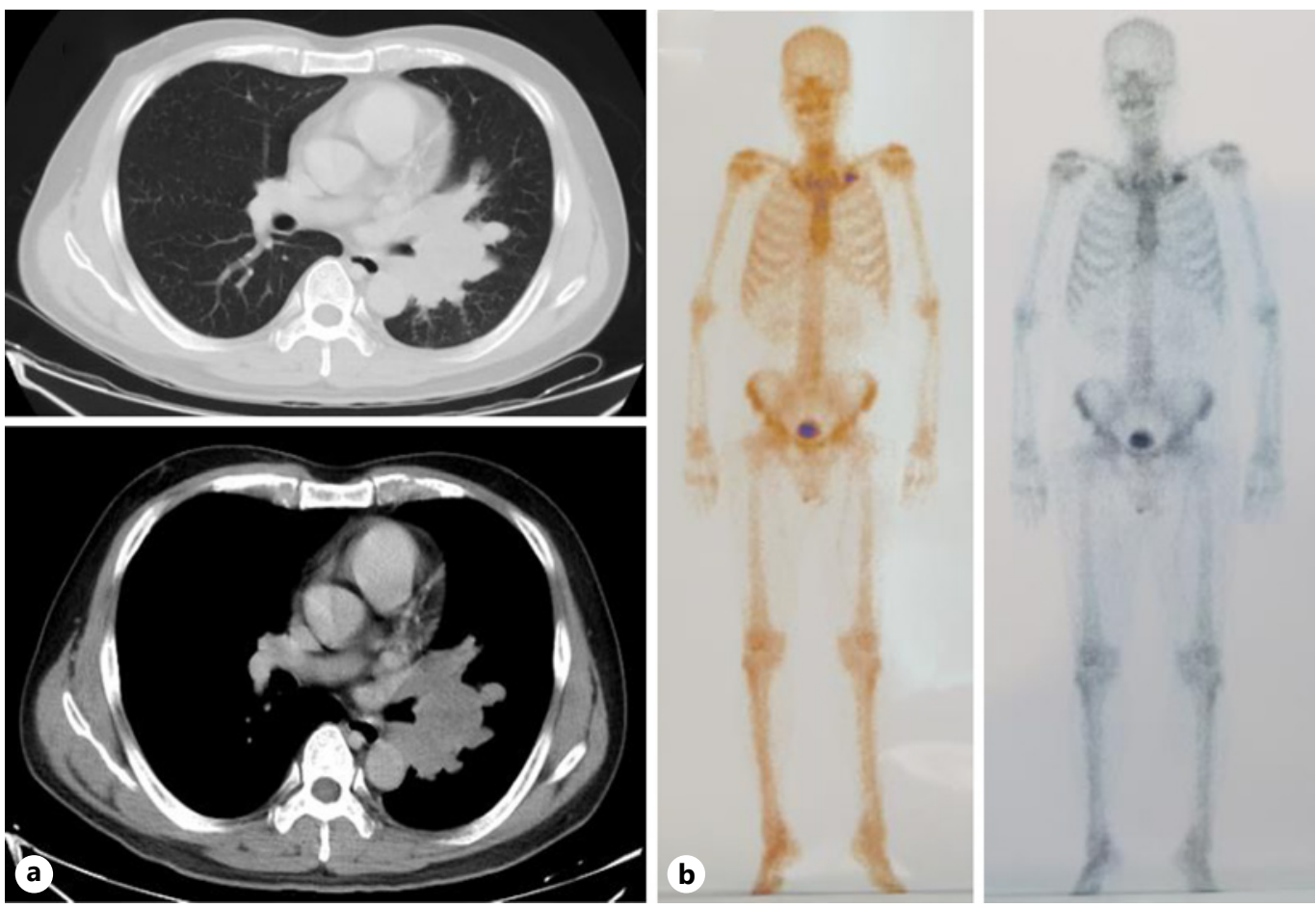

Fig. 2. Imaging results from initial hospitalization. a Chest CT shows a left hilum lung mass with enlargement of mediastinal and right hilum lymph nodes. b Bone scan shows an increased uptake in left second rib with high suspicion of metastasis. CT, computed tomography.
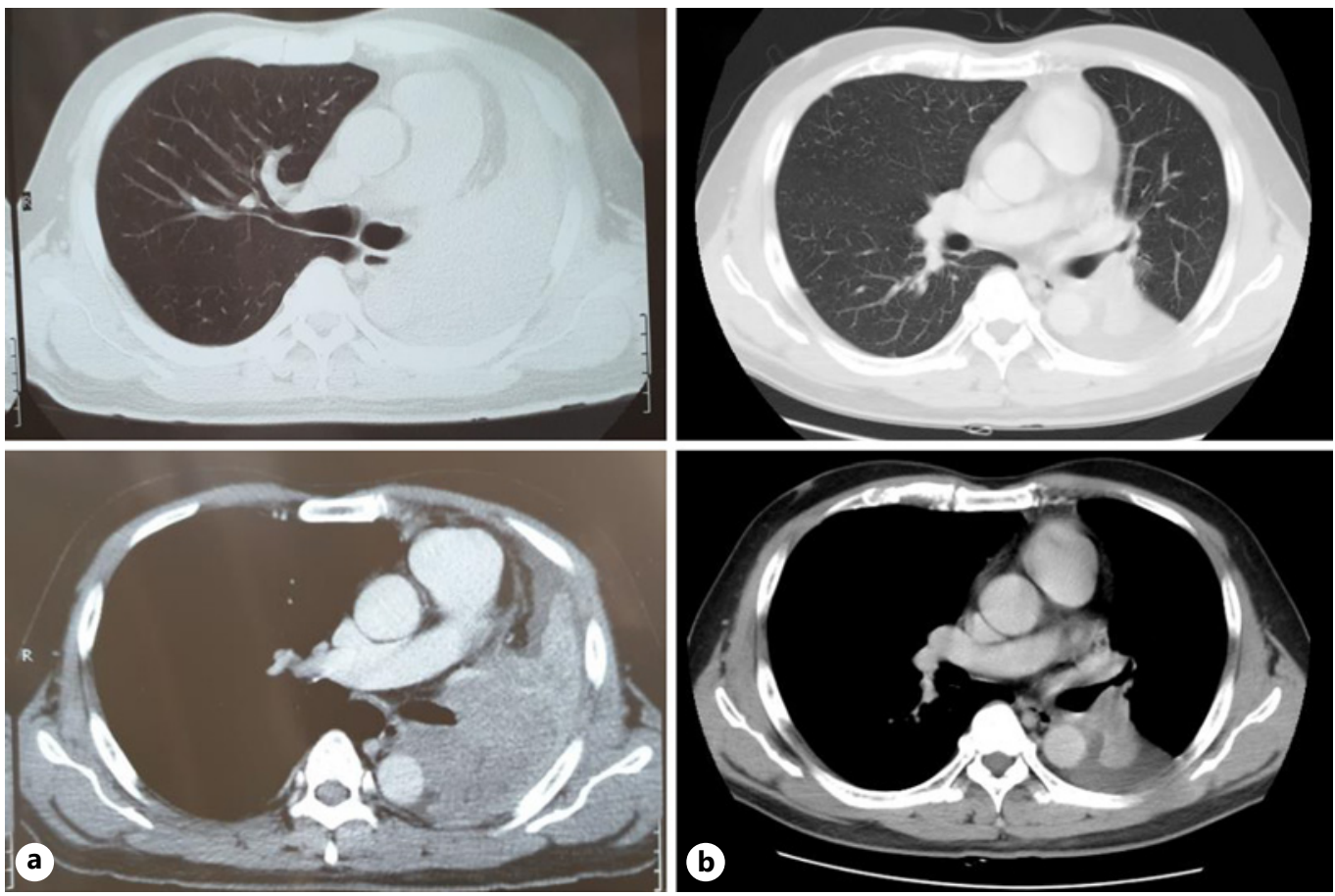

Fig. 3. Imaging results before and after the treatment of pembrolizumab. a Contrast-enhanced axial CT (after 3 cycles of paclitaxel) demonstrates complete atelectasis of the left lung due to a large left perihilar mass. b Image after 3 cycles of pembrolizumab show decreasing in size of left lung tumor to $34 \times 37 \mathrm{~mm}$ (compare with $88 \mathrm{~mm}$ before). CT, computed tomography. 


\section{Discussion and Conclusion}

Angiosarcoma is uncommon tumor that arises in the subcutaneous tissue of many sites of the body, typically head and neck, or breast. Angiosarcoma has a worse prognosis, with median overall survival are around 9 months [3]. In metastatic disease, standard treatment is based on chemotherapy with either doxorubicin or paclitaxel. However, chemotherapy rarely leads to complete and durable responses, challenging in clinical and oncology practice. Therefore, a novel therapeutic option is urgently required.

In the last 2 years, the advent of immunotherapy has provided a promising alternative for classical cytotoxic chemotherapy. Although these agents have been used in soft tissue sarcoma, the role of immunotherapy in angiosarcoma has just been reported in several case clinics [4-6]. Further, investigations will be needed to elucidate the efficacy and safety of this treatment.

Here, we had done a proof of concept for immunotherapy to halt the progression of lung tumor in pulmonary angiosarcoma. Our results open the new way to treat angiosarcoma. Early detection of programmed death ligand 1 (PD-L1) and early introduction of immunotherapeutic agents may be a promising strategy for angiosarcoma, especially for those with high PD-L1 expression.

\section{Statement of Ethics}

The written informed consent was obtained from the patient for publication of this case report and any accompanying images.

\section{Conflict of Interest Statement}

The authors declare that they have no conflicts of interest.

\section{Funding Sources}

There was no funding provided for the writing of this manuscript.

\section{Author Contributions}

All the authors equally contributed to writing and editing and agreed on submission of the final version. Quang T. Nguyen provided the study materials, collected and assembled the data, wrote the manuscript, and provided final approval and submission. Anh T. Pham provided the study materials, collected and assembled the data, wrote the manuscript, and provided final approval and submission. Thuy T. Nguyen provided the study materials, collected and assembled the data, wrote the manuscript, and provided final approval and submission. Tam T.T. Nguyen provided the patient, collected and assembled the data, and provided final approval and submission, Ky V. Le provided the pathologic images of the patient, collected and assembled the data, and provided final approval and submission.

\section{Karger'k}




\section{References}

1 Levy E, Korach A, Amir G, Milgalter E. Undifferentiated sarcoma of the pulmonary artery mimicking pulmonary thromboembolic disease. Heart Lung Circ. 2006 Feb;15(1):62-3.

2 Ren Y, Zhu M, Liu Y, Diao X, Zhang Y. Primary pulmonary angiosarcoma: three case reports and literature review. Thorac Cancer. 2016 Sep;7(5):607-13.

3 Young RJ, Brown NJ, Reed MW, Hughes D, Woll PJ. Angiosarcoma. Lancet Oncol. 2010 Oct 1;11(10):983-91.

4 Sindhu S, Gimber LH, Cranmer L, McBride A, Kraft AS. Angiosarcoma treated successfully with anti-PD-1 therapy: a case report. J Immunother Cancer. 2017 Jul 18 [cited 2021 Mar 15];5:58. Available from: https:// www.ncbi.nlm.nih.gov/pmc/articles/PMC5514460/.

5 Florou V, Rosenberg AE, Wieder E, Komanduri KV, Kolonias D, Uduman M, et al. Angiosarcoma patients treated with immune checkpoint inhibitors: a case series of seven patients from a single institution. J Immunother Cancer. 2019 Aug 8;7(1):213.

6 Hamacher R, Kämpfe D, Reuter-Jessen K, Pöttgen C, Podleska LE, Farzaliyev F, et al. Dramatic response of a PD-L1-positive advanced angiosarcoma of the scalp to pembrolizumab. JCO Precis Oncol. 2018 [cited 2021 Mar 15]. Available from: https://ascopubs.org/doi/full/10.1200/P0.17.00107. 